\title{
Quantum Wavefunction Annealing of Spin Glasses on Ladders
}

\author{
J. Rodríguez-Laguna \\ International School for Advanced Studies (SISSA), Via Beirut 2-4, I-34014 Trieste, Italy
}

(Dated: February 15, 2007)

\begin{abstract}
A technique inspired on quantum annealing is proposed in order to obtain the classical ground state of a spin-glass by tracking the full wavefunction of a given system within the subspace of matrix product states (MPS), using the density matrix renormalization group (DMRG). The technique is exemplified within the problem of obtention of the classical ground state of an Ising spin glass on ladder geometries. Its performance is evaluated and related to the entanglement entropy.

PACS numbers: $73.43 . \mathrm{Nq}, 05.10 . \mathrm{Cc} 75.40 . \mathrm{Mg}, 75.10 . \mathrm{Nr}$,
\end{abstract}

\section{INTRODUCTION}

Global optimization is one of the most challenging numerical tasks. Simulated thermal annealing (STA) has been, for more than twenty years, one of the most popular general purpose tools. Its strength relies on its ability to escape metastable minima via thermal fluctuations. Simulated quantum annealing $(\mathrm{SQA})^{\underline{1}}$ is a more recent algorithm which takes profit from quantum fluctuations for the same purpose. Both methods have a clear physical motivation: thermal annealing has been carried out by metallurgists for millenia. On the other hand, the first evidence of the superiority of quantum over thermal fluctuations in order to find the global minimum of a real physical system was only obtained in 1999, in the context of quantum spin glasses 2 .

Numerical implementations of SQA have relied heavily on the quantum-classical analogy described by the path integral Monte-Carlo method (PIMC). Standard simulated thermal annealing is applied to an enlarged system, which consists of several replicas of the original system in interaction. Slightly different versions of the method have succeeded in the obtention of the ground state of classical spin glasses 3.4 , atomic clusters ${ }^{5,6}$, traveling salesman problem ${ }^{7}$, kinetically constrained systems ${ }^{8}$ and small protein-folding problems ${ }^{5}$, among others. SQA has proved to work less efficiently than STA in other problems, such as 3-SAT $\underline{9}$ and some benchmark 1D potentials 10 .

Despite the success of the PIMC-SQA, there are reasons to look for different implementations $\underline{11}$. First of all, PIMC simulations must be carried out at finite temperature, with lower temperatures requiring a larger number or replicas and, therefore, higher computational cost. Also, some quantum systems suffer from the sign problem, or from problems with the Trotter break-up. Sampling difficulties are always a risk for annealing schemes, and ensuring ergodicity is often a highly non-trivial task.

Advancing in this line, Green's function Monte-Carlo technique was attempted by Stella and Santoro 2 . Its main disadvantage is the necessity of good trial variational wavefunctions. Real time evolution of the full wavefunction has been implemented by Suzuki and Okada 13 , making use of both exact methods and the time-dependent density matrix renormalization group (DMRG) algorithm 14. For systems where DMRG is efficient, loss of adiabaticity in the form of Landau-Zener level crossings is the main problem of this approach.

The proposal of this work is to perform the annealing on the full wavefunction, thus overcoming most of the difficulties associated with PIMC. But, as opposed to the Suzuki-Okada approach, a real time evolution is not needed either. Instead, the ground state of the full system is computed exactly for a high intensity of the quantum fluctuations. As these fluctuations are decreased, the ground state is subsequently updated, by making the necessary small modifications of the previous ground state. The full wavefunction of the ground state is stored in the form of a matrix product state (MPS), and is computed variationally using the DMRG.

As a benchmark case-study we have selected the obtention of the classical ground state of an Ising spinglass with couplings uniformly distributed in $[-1,1]$. The quantum fluctuations are provided by a transverse magnetic field. We have chosen the ladders for the underlying topology, since it is the simplest case which presents genuine frustration while retaining the quasi-1D character which is required for the DMRG to attain its maximum efficiency. Albeit the problem is in class $\mathrm{P}$, we will show it to be complex enough to be considered non-trivial.

This article is organized as follows. Section III introduces our model hamiltonian, summarizing its general features as applied to our featured topology. It is followed, in section III. by a detailed description of the proposed quantum wavefunction annealing (QWA) method. In section IV we analyze the results of the numerical experiments we have conducted. We conclude, in section [V with a discussion of the advantages and limits of the method, and its possible extensions.

\section{RANDOM ISING MODEL IN A TRANSVERSE FIELD ON LADDERS}

Our model hamiltonian is the random Ising model in a transverse field (RITF), which we define on an arbitrary graph $\mathcal{G}$ with $N$ sites 15 : 


$$
H=-\sum_{\langle i, j\rangle} J_{i j} \sigma_{i}^{z} \sigma_{j}^{z}-\Gamma \sum_{i} \sigma_{i}^{x}-\sum_{i} h_{i}^{z} \sigma_{i}^{z}
$$

where $\langle i, j\rangle$ denotes nearest neighbours in $\mathcal{G}$, the $J_{i, j}$ are independent random variables uniformly distributed in $[-1,1], \Gamma$ is the transverse field and $\sigma_{i}^{x}$ and $\sigma_{i}^{z}$ are Pauli matrices at site $i$. We will work on the basis of eigenstates of $\sigma^{z}$. Unless explicitly stated, we will assume the longitudinal fields $h_{i}^{z}$ to be zero. The first term in eq. 1 may be considered as a potential energy, and the second as a kinetic term: the magnitude of $\Gamma$ measures the intensity of the quantum fluctuations. For $\Gamma=0$, the ground state is composed of only two states, related by a trivial $+\Leftrightarrow-$ symmetry, which we term the classical ground states (CGS) of the spin glass. As $\Gamma \rightarrow \infty$, on the other hand, all spins should be pointing in the $X$-direction, therefore obtaining the following ground state:

$$
|X\rangle=\otimes_{i=1}^{N} \frac{1}{\sqrt{2}}\left(|+\rangle_{i}+|-\rangle_{i}\right)
$$

All the wavefunction components of the ground state take the same value in our basis for $\Gamma \rightarrow \infty$.

The behaviour of this system at $T=0$ is rather well known in $1 \mathrm{D}^{16,17}$ thanks to an insightful RG analysis, in $2 \mathrm{D}^{18}$ and $3 \mathrm{D}^{19}$ using quantum Monte-Carlo, and in random graphs with fixed connectivity $\underline{20}$ making use of the DMRG. In all these cases, the system presents a quantum spin-glass transition (QSGT) at a finite value of $\Gamma=\Gamma_{c}$, where the energy gap vanishes. Above that value, the system is said to be in a quantum paramagnetic regime, whilst below it behaves as a quantum spin-glass.

The problem of finding the global CGS for equation 1 at $\Gamma=0$ is known to be in class $\mathrm{P}$ for $D=1$ and for $D=2$ in the absence of longitudinal magnetic field $h^{z}$. In the $2 \mathrm{D}$ case with $h^{z} \neq 0$, the $3 \mathrm{D} \operatorname{case}^{21}$ or in random graphs with fixed connectivity $\underline{22}$, the problem is NP-complete.

Rectangular ladders constitute the simpest topology in which the system described by equation 1 presents genuine frustration. Their size is characterized by two numbers: $L \times w$, where $L$ is the length and $w$ is their width, or number of legs. Figure 1 shows a specimen with size $5 \times 2$. The classical spin-glass with $\pm J$ couplings on these ladders has been analyzed by a number of authors 23.24 . We will now discuss the complexity of the energy landscape for $\Gamma=0$ and a few basic characteristics of the nature of the QSGT.

Minimization of the classical hamiltonian $(\Gamma=0)$ can be done using STA or PIMC-SQA. For the system sizes under consideration, both methods yield robust estimates for the CGS energy following the schemes described by Santoro and coworkers 4 . In order to analyse the complexity of the energy landscape, we relax the parameters

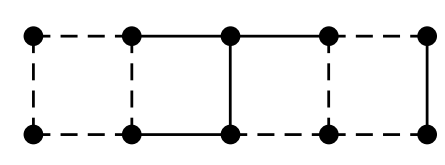

FIG. 1: A rectangular ladder of dimension $5 \times 2$. Coupling constants $J_{i j}$ are associated to links of the graph. In the example of the figure, dashed (continuous) lines represent negativeAFM (positive-FM) links, and the system is frustrated.

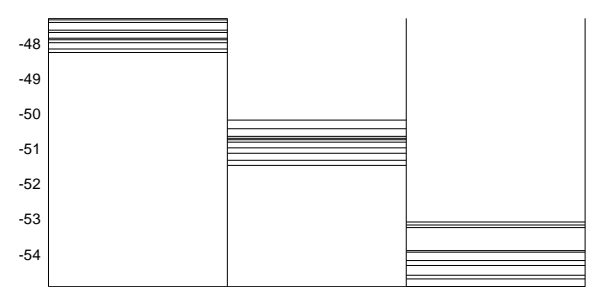

FIG. 2: Some local minima of the classical hamiltonian for three $40 \times 2$ samples, obtained with a non-robust STA in order to sample the low energy configurations.

until the system becomes non-robust, i.e.: it yields different results in different runs. At this point, the annealing processes provide us a series of sample configurations which constitute local minima of the energy. With this purpose, we have applied a STA algorithm with a multiplicative scheme for $\beta$, i.e.: $\beta \rightarrow r \beta$, from $\beta_{0}=0.1$ to $\beta_{\max }=10^{6}$, with $r=1+10^{-5}$ and $10^{4}$ steps per temperature. The results for three samples of a $40 \times 2$ ladder are shown in figure 2. Each column contains the energy values obtained after the procedure was repeated 20 times, providing several different local energy minima (about 10). In all the cases shown, the lowest energy corresponds to the CGS. This result points to a complex energy landscape for the spin-glass ladders.

The energy per spin of the CGS seems to converge, in the case of the 2-legged ladders, to $\epsilon^{(2)} \approx-0.64$. In absence of frustration, this value would be -0.75 . In the case of the ladder with 4 legs, it converges to $\epsilon^{(4)} \approx$ -0.71 , with the unfrustrated value being -0.875 .

At $T=0$, the system described by eq. 1 presents a quantum spin-glass transition (QSGT) at a finite value of $\Gamma=\Gamma_{c}$. The finite-size DMRG algorithm, suitably adapted for our case $\mathrm{e}^{20}$, can be used to characterize this transition. The behavior of some relevant observables has been traced in figure 3; (a) the energy gap $\Delta E$, (b) the maximum block entropy $S_{\max }$, given by

$$
S_{\text {max }} \equiv-\operatorname{Tr} \rho_{L} \ln \left(\rho_{L}\right)
$$

where $\rho_{L}$ is the reduced density matrix for the left half of the ladder, and (c) the spin glass susceptibility $\chi_{S G}$, as defined by the following formula: 


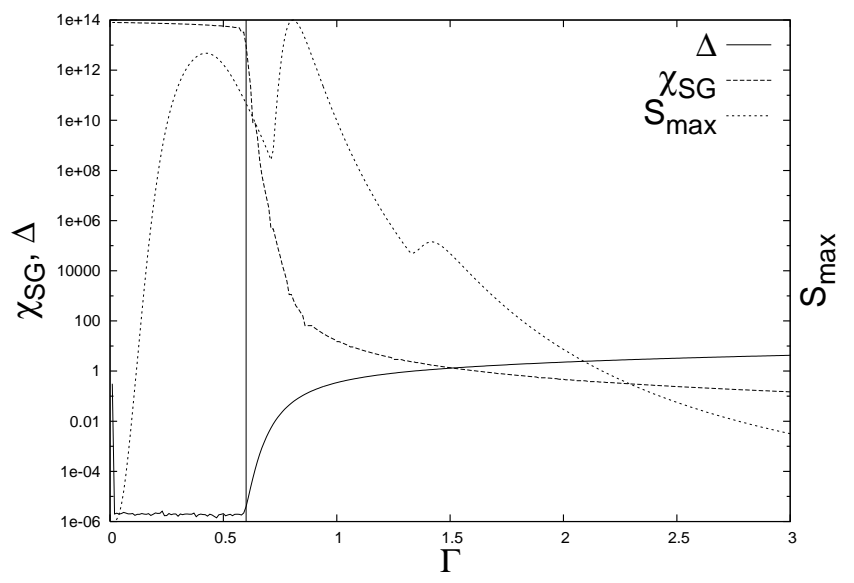

FIG. 3: Behavior of the energy gap $\Delta E$, the maximum block entropy $S_{\max }$ and the spin-glass susceptibility $\chi_{S G}$ for a sample spin-glass ladder of dimension $40 \times 2$, as a function of $\Gamma$. The QSGT is marked very clearly by both $\chi_{S G}$ and $\Delta E$. On the other hand, $S_{\max }$ seems to have a more erratic behavior.

$$
\chi_{S G} \equiv \frac{1}{N} \sum_{i, j} \lim _{h_{j}^{z} \rightarrow 0}\left(\frac{\left\langle\sigma_{i}^{z}\right\rangle}{h_{j}^{z}}\right)^{2}
$$

i.e.: a small longitudinal magnetic field $h_{j}^{z}$, applied at site $j$, generates a magnetization response on each site $i$, which is measured (and squared, so as to disregard its sign); the results are summed over all sites $i$ and averaged over all sites $j$. The value of $\Gamma_{c}$ changes from sample to sample. Within a single sample, $\Delta E$ vanishes and $\chi_{S G}$ diverges at the same value of $\Gamma_{c}$, as it is shown in figure 3. For that sample, $\Gamma_{c}=0.6$. The entropy is seen to present a more complex behavior.

Some insight can be gained by tracing a few individual components of the full wavefunction, which is possible within the DMRG framework ${ }^{20}$. Figure 4 shows that, well within the paramagnetic regime, all wavefunction components take the same value. As we decrease $\Gamma$, the configurations with low energy start to increase its weight in the ground state wavefunction, while the configurations with high energy start to decrease. At the critical point, all the configurations have started their decay, except the one with the lowest energy.

More theoretical and analytical work is needed in order to fully characterize this QSGT. Some comments about it are provided in section $\mathrm{V}$. This preliminary analysis can be summarized as: (a) the energy landscape of the classical problem is complex, and (b) there is a quantum phase transition at a finite value of $\Gamma$.

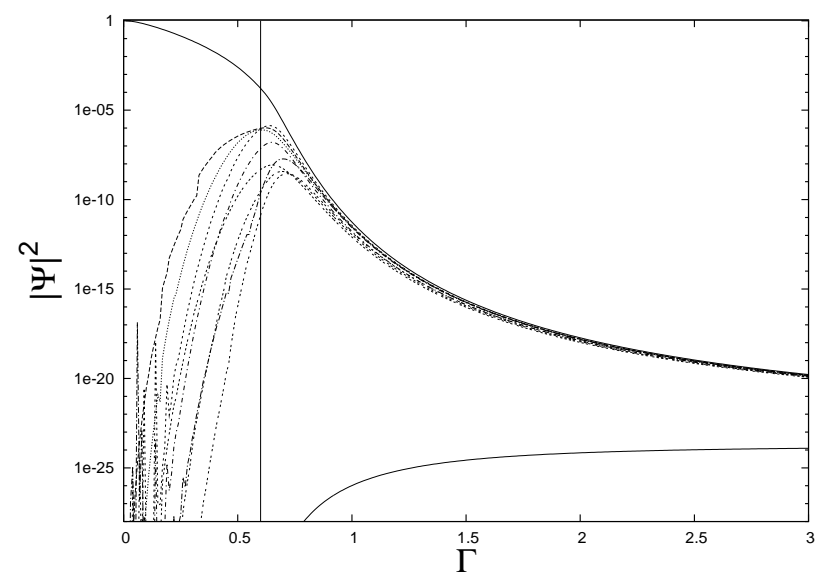

FIG. 4: Wavefunction components for some selected configurations, as a function of $\Gamma$, for a sample ladder with size $40 \times 2$. All the configurations but one are local minima of the classical energy function, obtained using a non-robust STA. The transition point is marked with an vertical line.

\section{QUANTUM WAVEFUNCTION ANNEALING OF SPIN GLASS SYSTEMS}

Quantum wavefunction annealing (QWA) is an implementation of simulated quantum annealing (SQA) which does not require real time evolution, configurations sampling or Trotter splitting. It makes extensive use of matrix product states (MPS) 25,26 and the density matrix renormalization group (DMRG) 27 . For the benefit of the readers which are not acquainted with those techniques, the first paragraph of this section describes a simpler formulation based on the Lanczos algorithm, but which can only be used for very small lattices.

The Lanczos diagonalization method improves its performance dramatically if a good seed to the real ground state of the system is provided 28 . The hamiltonian given by equation 1 has a simple behaviour for $\Gamma \rightarrow \infty$ : its ground state is known to be given by the state $|X\rangle$ defined in equation 2. Therefore, starting with such a state as a seed, it is fairly easy for the Lanczos algorithm to obtain the exact ground state for a finite but high value $\Gamma_{0}$. This value may be decreased at small finite steps, $\Gamma \rightarrow \Gamma-\Delta \Gamma$, and the ground state for the previous value of $\Gamma$ can be employed as the seed for the computation of the ground state at the new value. In quantum annealing, the adiabatic theorem is needed in order to prove that convergence to the CGS is sure in the limit $\Delta \Gamma \rightarrow 0$. In our case, a weaker result is enough: convergence is sure as long as there is a finite overlap between the ground states at any two consecutive values of $\Gamma$.

The obvious drawback of the previous algorithm is the size of the Hilbert space, $\operatorname{dim}(\mathcal{H})=2^{N}$. Using the plain Lanczos algorithm, all the wavefunction components should be stored and acted upon. A solution is to choose a low-dimensional subspace which is known to contain the ground state of the hamiltonian given by 
equation 1 for all values of $\Gamma$. Matrix product states (MPS) can provide such a subspace in some cases. These states may be written down as:

$$
|\Psi\rangle=\sum_{i} \operatorname{tr}\left(A^{s_{1}} A^{s_{2}} \cdots A^{s_{N}}\right)\left|s_{1}, s_{2}, \cdots, s_{N}\right\rangle
$$

where $\left|s_{1}, s_{2}, \cdots, s_{N}\right\rangle$ are the eigenstates of $\sigma_{i}^{z}$ and the $A^{s_{i}}$ are $2 N$ matrices of dimension $m \times m$, whose entries are the variational parameters of our Ansatz. The dimension of the MPS subspace is, therefore, bounded by $2 \mathrm{Nm}^{2}$. Both the computational cost and the accuracy of the method depend strongly on the dimension $m$ of the matrices, whose physical meaning is the following: for all possible left-right splittings of the system, the ground state is approximated retaining $m$ states to represent the left part and other $m$ states for the right part. For any possible state of the system, its representation as an MPS becomes exact for $m$ large enough. Of course, the dimensions $m$ can be made local, $m_{i}$, if necessary.

The DMRG is a variational method within the subspace of the MPS 29 . It profits from the use of a density matrix in order to select the $m$ states for the left and right blocks which fit best to our current approximation of the global ground state. The neglected eigenvalues of the density matrix provide a way to monitor the accuracy of the method. In all our applications, the tolerance to the total neglected probabilities is fixed beforehand to a certain value $\eta$, and the number of retained states $m(\eta)$, is adapted in consonance.

The MPS represent faithfully ground states of local 1D hamiltonians 30 . In other cases, at this stage, only educated experience can decide whether they are appropriate or not. They are specially suited to $1 \mathrm{D}$ and quasi-1D problems (e.g. ladders, trees), although natural extensions to higher dimensions are in active development ${ }^{31,32}$. A crucial tool is von Neumann's block entropy, $S=$ $-\operatorname{tr}\left(\rho \log _{2} \rho\right)$. In crude terms, $m$ should scale as $\exp (S)$ in order to obtain an accurate DMRG method. In 1D, the entropy $S$ is known to be bounded for non-critical systems and to scale as $\log (N)$ for a critical one ${ }^{33}$. For higher dimensions, the area law predicts the entropy to scale as $L^{d-1}$ out of criticality, where $L$ is a typical dimension of the system 34 .

As in the Lanczos case, the DMRG can benefit from a good seed when searching the ground state of a hamiltonian through the use of the wavefunction transformations ${ }^{35}$, which allow to use the solution of an RG-step as an initial step for the next one. Having found the ground state of a certain hamiltonian $H$, a few finitesize sweeps will adapt it to become the ground state of a slightly modified hamiltonian $H^{\prime}$. In normal cases, this procedure is not needed, since the number of sweeps required for convergence to the ground state for any hamiltonian is small. It may become very useful when (a) it is known that the ground state of a certain hamiltonian can be represented as a MPS with low $m$ but (b) the probability of the DMRG getting stuck at an excited state is very high. This is the case for the ground state of 1 in the quantum spin-glass phase 20 .

Our proposed QWA algorithm is, therefore, the following one:

(a) The ground state of the system 1 is obtained for very high $\Gamma$.

(b) The transverse field $\Gamma$ is decreased $\Gamma \rightarrow \Gamma-\Delta \Gamma$.

(c) A few finite-size sweeps of the DMRG are carried out, which adapt the ground state to the current value of $\Gamma$.

(d) Go to (b) if $\Gamma$ is not yet zero.

(e) Measure the energy and classical ground state.

This approach is deterministic, i.e.: not limited by sampling problems. It works directly with the quantum hamiltonian, therefore does not require any Trotter break-up, and works directly at $T=0$. Since it does not simulate real time evolution, it is not prone to Landau-Zener level-crossings. Loss of adiabaticity is not, therefore, a crucial issue. As it was stated, it is enough to ensure that the overlap between the ground states at consecutive values of $\Gamma$ is finite. A high value of $\Delta \Gamma$ is allowed far away from the quantum phase transition, while a more reduced value will be taken near it. Our precise adaptive reduction schedule is described in the following section.

On the negative side, it is based on a method which is specially designed for $1 \mathrm{D}$ systems. We will say more about this in the conclusions. Its main weakness stems from the inability of the MPS to represent faithfully the ground state for all values of $\Gamma$ for higher dimension. The efficiency and practical issues of the implementation are analyzed in the next section.

\section{NUMERICAL EXPERIMENTS}

In order to study the applicability of QWA, we have generated random samples of ladders with $w=2$ and $w=4$ legs, and lengths ranging from $L=20$ to $L=160$. We have also considered random graphs of fixed connectivity $K=3$ and, as a check, we have also analysed linear chains (although the optimization problem is trivial in that case). The annealing scheme has always been the same: starting with $\Gamma_{0}=3$ and using an adaptative reduction, $\Gamma \rightarrow \Gamma-\Delta \Gamma$ with

$$
\Delta \Gamma=\min (0.5,0.1 / S)
$$

where $S$ is the maximum block entropy for the previous value of $\Gamma$. This way we ensure that, near the QSGT when the entropy is higher- the steps are shorter. We insist in this fact: the adiabatic theorem and Landau-Zener level crossings are not the limitations of this method. 
Therefore, a decrease in the size of the steps does not necessarily increase the probability of success.

The last annealing step was always taken with $\Gamma_{\min }=$ 0.01 , a value which is low enough for practical purposes. The obtention of the minimum energy configuration was carried out measuring the $z$-component of the spin polarization of all sites after convergence for $\Gamma=\Gamma_{\min }$. Of course, due to the obvious symmetry $+\Leftrightarrow-$, the expectation value for each of these components is zero. We have used a common procedure in quantum spin-glass calculations: the insertion of a very small longitudinal magnetic field $h^{z}=10^{-6}$ in a single site, selected at random, in order to break the symmetry. In the QSG phase, because of the divergence of the spin-glass susceptibility discussed above, an infinitesimal localized magnetic field polarizes the full sample.

Table 1 provides the basic set of results. For each geometry we have selected 20 samples and performed the QWA algorithm on them. We have also chosen different values of the neglected probabilities tolerance, $\eta$. For each run, QWA is said to obtain a success if its minimum energy is equal to the value obtained by STA and PIMC-SQA. QWA has had success, in all the attempted geometries, whenever $\eta=10^{-8}$. When the tolerance was decreased, the CGS was missed with higher probability, although the method can be seen to be rather robust for spin-glass ladders, since the tolerance must be raised up to $10^{-3}$ in order to decrease the probability of success to $50 \%$.

We have also tried to check the method with a different graph topology: random graphs with fixed connectivity, $K=3$. For $N=20$ sites, employing a tolerance of $\eta=10^{-8}$, the method provides again $100 \%$ of success. If the size is increased, that tolerance can not be set, since the number of states $m$ to be kept becomes prohibitive. Therefore, we have carried out the experiments with $\eta=$ $10^{-6}$ for a $N=100$ sample, and the probability of success reduces to $45 \%$.

The time for the QWA algorithm scales as a power law of the system size: $T \approx L^{\alpha}$. For linear chains, using the results from table $1 \alpha \approx 1.3 \pm 0.1$. For 2-legged ladders, $\alpha \approx 2 \pm 0.1$. In the case of the 4-legged ladders, the power law fit has a higher errorbar, with a surprising exponent of $\alpha \approx 1.5 \pm 0.2$.

\section{A. QWA and block entropy}

This polynomial growth can be theoretically explained. For quasi-1D systems at criticality, the von Neumann entropy grows as a logarithm of the system size $33, S_{c}(L) \approx$ $a \log (L)+b$. The value of $a$ has been related, in some random systems, to the central charge of the associated conformal field theory ${ }^{36}$. On the other hand, the maximum number of retained states scales as the exponential of the entropy, $m \approx \exp (S)$, and the time for a DMRG sweep scales as $T \approx L m^{2}$. The most expensive DMRG sweep for a QWA simulation takes place at the critical point,

\begin{tabular}{lccc}
\hline \hline Geom. & $\eta$ & Success & Time \\
20 & $10^{-8}$ & $100 \%$ & $1.2 \pm 0.2$ \\
40 & $10^{-8}$ & $100 \%$ & $3.9 \pm 0.8$ \\
80 & $10^{-8}$ & $100 \%$ & $10 \pm 2$ \\
160 & $10^{-8}$ & $100 \%$ & $21 \pm 4$ \\
320 & $10^{-8}$ & $100 \%$ & $56 \pm 10$ \\
\hline $20 \times 2$ & $10^{-8}$ & $100 \%$ & $17 \pm 8$ \\
$40 \times 2$ & $10^{-8}$ & $100 \%$ & $60 \pm 15$ \\
$80 \times 2$ & $10^{-8}$ & $100 \%$ & $240 \pm 30$ \\
$160 \times 2$ & $10^{-8}$ & $100 \%$ & $600 \pm 110$ \\
\hline $40 \times 2$ & $10^{-5}$ & $80 \%$ & $26 \pm 3$ \\
$40 \times 2$ & $10^{-3}$ & $50 \%$ & $13 \pm 1$ \\
\hline $20 \times 4$ & $10^{-8}$ & $100 \%$ & $1600 \pm 600$ \\
$40 \times 4$ & $10^{-8}$ & $100 \%$ & $6800 \pm 2000$ \\
$80 \times 4$ & $10^{-8}$ & $100 \%$ & $14000 \pm 3000$ \\
$160 \times 4$ & $10^{-8}$ & $100 \%$ & $27000 \pm 3000$ \\
\hline $\mathrm{RG}-20$ & $10^{-8}$ & $100 \%$ & $190 \pm 150$ \\
$\mathrm{RG}-100$ & $10^{-6}$ & $45 \%$ & $15000 \pm 7000$ \\
\hline \hline
\end{tabular}

TABLE I: Numerical results for the QWA method. The first column states the geometry of the system under study: $L$ if it is a linear chain, $L \times w$ if it is a ladder, and RG- $N$ if it is a random graph with connectivity $K=3$. The second gives the tolerance for neglected probabilities in the DMRG, $\eta$. The third provides the percentage of success. The fourth column provides the average time (in seconds) for the QWA of a single sample.

and therefore we may expect $T \approx L \exp \left(2 S_{c}\right) \approx L^{2 a+1}$. Thus, the theoretical prediction is $\alpha \simeq 2 a+1$.

In the linear chain case, the entropy at criticality was predicted by Refael and Moore ${ }^{36}$ to have a coefficient $a=\ln (2) / 6 \approx 0.11$, in agreement with our own numerical measurements. Therefore, the theoretical prediction for the $\alpha$ exponent is $\approx 1.22$, which is not far from the $1.3 \pm 0.1$ obtained numerically. In the case of the 2-legged ladder, there is no theoretical estimate, but our own numerical simulations provide a value $a \approx 0.55 \pm 0.1$, thus giving an estimate for $\alpha$ around 2.1, in agreement with the numerically observed value $\alpha \approx 2 \pm 0.1$.

\section{CONCLUSIONS AND FURTHER WORK}

In this paper we have introduced a method for the obtention of the minimum energy configuration of a spin-glass, based on the annealing of the full wavefunction represented as a matrix product state (MPS) using the density matrix renormalization group (DMRG). The method has been termed quantum wavefunction annealing (QWA), and we have assessed and quantified its efficiency, by comparing its results with those provided by other robust methods. For spin-glass ladders of 2 and 4 legs with lengths ranging from 20 to 160, the method has 
always provided the optimum solution when the probability tolerance is set to $\eta=10^{-8}$. The running time scales as $L^{2}$ for 2-legged ladders, and, surprisingly, as $L^{1.5}$ for 4 -legged ones. This anomaly requires further clarification, perhaps in the line of thought of Ferraro et $\mathrm{a} \mathrm{l}^{37}$. For random graphs with fixed connectivity $K=3$, on the other hand, the tolerance has to be increased in practice to $10^{-6}$, and the probability of success falls to $45 \%$ for $N=100$.

The most crucial parameter which determines the success of the method is the probability tolerance $\eta$. Whenever we were able to set $\eta=10^{-8}$, for whichever topology, the system always attained the optimum configuration. Unfortunately, for topologies which are not quasi-1D, the number of retained states in DMRG, $m$, grows very fast when the tolerance is decreased. Other parameters, such as the annealing velocity, do not have such a direct relevance to the quality of the results. Loss of adiabaticity is not the main issue for this method: a finite overlap between the ground state wavefunctions at consecutive annealing steps is enough to ensure convergence, as long as the number of retained states $m$ is large enough for our Ansatz wavefunction to represent both of them faithfully.

Therefore, it seems reasonable to think that QWA can be converted into a method to find the optimum configuration of all quasi-1D systems in polynomial time. This suggests that, in fact, no quasi-1D problem can be NP-complete. On the other hand, for problems which are known to be NP-complete, such as the random Ising spin-glass in a 2D lattice with a longitudinal field, or on random graphs of fixed connectivity, the time for an algo- rithm which is able to obtain the optimum with certainty should scale exponentially with the size of the system.

This fact puts a limit to possible extensions of the QWA algorithm described in this paper. The natural extension of the MPS are the tensor product states (TPS) analyzed by Nishino and coworkers ${ }^{31}$ or the pairentangled particle states (PEPS) ${ }^{32}$ of Verstraete and Cirac. The computational power of this type of states has been analyzed recently $\stackrel{38}{ }$, pointing to the fact that any attempt to solve NP-complete problems with an exact QWA algorithm using these states would require an exponential time, which is compatible with the usually believed notion that $\mathrm{P} \neq \mathrm{NP}$.

The most promising lines for future work are, therefore, in the development of heuristic algorithms, running in polynomial time, which may give the absolute minimum energy state with high probability for some problems. A possibility is the development of a QWA algorithm using TPS (or PEPS) with a fixed number of retained states $m$. Another possibility is to use the DMRG approach to non-equilibrium classical problems $\underline{39}$ to develop a classical wavefunction annealing algorithm, which would solve the Fokker-Planck equation associated to simulated thermal annealing.

\section{Acknowledgments}

The author acknowledges G. Santoro, A. Trombettoni, M.A. Martín-Delgado and G. Sierra for very useful discussions.
1 A. Das and B. K. Chakrabarti, Quantum Annealing and Related Optimization Methods, Lecture Notes in Physics (Springer Verlag, 2005).

2 J. Brooke, D. Bitko, T. F. Rosenbaum, and G. Aeppli, Science 284, 779 (1999).

3 T. Kadowaki and H. Nishimori, Phys. Rev. E 58, 5355 (1998).

4 G. E. Santoro, R. Martoňák, E. Tosatti, and R. Car, Science 295, 2427 (2002).

5 Y.-H. Lee and B. Berne, J. Phys. Chem. A 104, 86 (2000).

6 T. Gregor and R. Car, Chem. Phys. Lett. 412, 125 (2005).

7 R. Martoňák, G. E. Santoro, and E. Tosatti, Phys. Rev. E 70, 057701 (2004).

8 A. Das, B. Chakrabarti, and R. B. Stinchcombe, Phys. Rev. E 72, 026701 (2005).

9 D. A. Battaglia, G. E. Santoro, and E. Tosatti, Phys. Rev. E 71, 066707 (2005).

10 L. Stella, G. E. Santoro, and E. Tosatti, Phys. Rev. B 72, 014303 (2005).

11 D. Battaglia, L. Stella, O. Zagordi, G. E. Santoro, and E. Tosatti, in Quantum annealing and related optimization methods, edited by A. Das and B. K. Chakrabarti (Springer Verlag, 2005).

12 L. Stella and G. E. Santoro, cond-mat/0608420 (2006).

13 S. Suzuki and M. Okada, in Quantum annealing and re- lated optimization methods, edited by A. Das and B. K. Chakrabarti (Springer Verlag, 2005).

14 S. R. White and A. E. Feiguin, Phys. Rev. Lett. 93, 076401 (2004).

15 B. K. Chakrabarti, A. Dutta, and P. Sen, Quantum Ising phases and transitions in transverse Ising models, Lecture Notes in Physics (Springer Verlag, 1996).

16 D. S. Fisher, Phys. Rev. B 51, 6411 (1995).

17 D. S. Fisher and A. P. Young, Phys. Rev. B 58, 9131 (1998).

18 H. Rieger and A. P. Young, Phys. Rev. Lett. 72, 4141 (1994).

19 M. Guo, R. N. Bhatt, and D. A. Huse, Phys. Rev. Lett. 72, 4137 (1994).

20 J. Rodríguez-Laguna and G. E. Santoro, condmat/0610661 (2006), submitted to PRB.

21 F. Barahona, J. Phys. A: Math. Gen. 15, 3241 (1982).

22 F. Liers, M. Palassini, A. K. Hartmann, and M. Jünger, Phys. Rev. B 68, 094406 (2003).

23 D. C. Mattis and P. Paul, Phys. Rev. Lett. 83, 3733 (1999).

${ }^{24}$ T. Uda, H. Yoshino, and H. Kawamura, Phys. Rev. B 72, 024442 (2005).

25 M. Fannes, B. Nachtergaele, and R. Werner, Commun. Math. Phys 144, 443 (1992).

26 J. Román, G. Sierra, J. Dukelsky, and M. Martín-Delgado, 
J. Phys. A: Math. Gen. 31, 9729 (1998).

27 S. R. White, Phys. Rev. Lett. 69, 2863 (1992).

${ }^{28}$ G. H. Golub and C. F. van Loan, Matrix Computations (Hopkins University Press, 1996).

29 S. Rommer and S. Östlund, Phys. Rev. B 55, 2164 (1997).

${ }^{30}$ F. Verstraete and J. I. Cirac, Phys. Rev. B 73, 094423 (2006).

31 T. Nishino, Y. Hieida, K. Okunishi, N. Maeshima, and Y. Akutsu, Prog. Theor. Phys. 105, 409 (2001).

${ }^{32}$ F. Verstraete and J. Cirac, cond-mat/0407066 (2004).

33 G. Vidal, J. I. Latorre, E. Rico, and A. Kitaev, Phys. Rev. Lett. 90, 227902 (2002).
34 M. Sredniki, Phys. Rev. Lett. 71, 666 (1993).

35 S. R. White, Phys. Rev. Lett. 77, 3633 (1996).

36 G. Refael and J. Moore, Phys. Rev. Lett. 93, 260602 (2004).

37 A Ferraro, A. Garcia-Saez and A. Acin, quant-ph/0701009 (2007).

38 N. Schuch, M. M. Wolf, F. Verstraete, and J. I. Cirac, quant-ph/0611050 (2006).

39 A. Degenhard, J. Rodriguez-Laguna, and S. N. Santalla, Mult. Model. Simul. (SIAM) 3, 89 (2004). 